\title{
Desenvolvimento e Avaliação de Material Web para o Ensino de Decimais Utilizando Exemplos Incorretos
}

\author{
Seiji Isotani - Universidade de São Paulo (ICMC-USP) - sisotani@icmc.usp.br \\ Bruce M. McLaren - Carnegie Mellon University - bmclaren@cs.cmu.edu
}

\begin{abstract}
Resumo. $O$ uso de exemplos incorretos como ferramenta pedagógica ainda é um assunto controverso. Muitos professores são ambivalentes e, até mesmo, hostis quanto ao seu uso. No ensino de matemática, este sentimento persiste. Poucos professores ensinam utilizando exemplos incorretos. Contudo, pesquisadores na área de educação matemática vêm demonstrando que a discussão de conceitos utilizando erros incentiva a reflexão e a construção do conhecimento. Devido a esta polêmica, existe uma lacuna de material didático que utiliza esta abordagem. Dessa forma, este trabalho possui dois objetivos principais. O primeiro é desenvolver material Web que utiliza exemplos incorretos para ensinar matemática; com foco no conceito de decimais. $O$ segundo é investigar os benefícios (ou malefícios) deste material. Foi conduzido um estudo com 429 alunos do ensino fundamental de duas escolas públicas dos EUA para avaliar o impacto deste material na aprendizagem de decimais. Os resultados indicam um significativo aumento na aprendizagem dos alunos, dando mais evidências a favor do uso de exemplos incorretos para ajudar a aprendizagem de matemática.
\end{abstract}

Palavras-chave: exemplos incorretos, aprendizagem de matemática, decimais

\section{Development and Evaluation of Web-based Materials for Teaching Decimals Using Erroneous Examples}

\begin{abstract}
The use of erroneous examples as a pedagogical tool is still a controversial issue. Many teachers are ambivalent and even hostile about the use of these examples during the learning process. In the field of mathematics education, this feeling persists. Few teachers use erroneous examples in their classes. However, researchers in the field have shown that the discussion of concepts using errors encourages student's critical thinking, reflection and knowledge construction. Because of such controversy, there is a lack of educational content that uses erroneous examples. Thus, this work has two main objectives. First, the development of Web content that uses erroneous examples to teach math in the domain of decimals. Second, investigate the benefits (or harms) of this material. We conducted a study with 429 students from two public schools in the US to evaluate the impact of using such material. The results indicate a significant increase in performance on student learning, providing more evidence in favor of erroneous examples as a good educational tool to teach mathematics.
\end{abstract}

Keywords: erronenous examples, math learning, decimals 


\section{Introdução}

Análises de livros didáticos de matemática em países do ocidente (e.g. Estados Unidos EUA) revelam que o conteúdo tende a se concentrar excessivamente na fluência de procedimentos (resolver sem pensar), dando menos importância à compreensão conceitual do tópico abordado (Mayer, Sims, e Tajika, 1995). Assim, grande parte dos alunos do ocidente realizam adequadamente a aplicação de procedimentos para resolver problemas simples, mas apresentam dificuldades durante a resolução de problemas de matemática que exigem o uso de estratégias avançadas (Mullis, Martin Gonzalez, e Chrostowski, 2004). Como resultado, as recentes avaliações feitas por orgãos internacionais, indicam que os alunos das escolas ocidentais (e.g. Brasil e EUA) possuem grandes problemas de aprendizagem. Em 2010, a avaliação internacional realizada pela OECD conhecida como PISA (Programme for International Student Assessment) indicou que alunos do Brasil e dos EUA têm índices inadequados de aprendizagem de matemática.

Um dos tópicos nos quais os alunos demonstraram grandes dificuldades foi números decimais. Este problema não é recente. Está bem documentado que os alunos do ensino fundamental têm grandes dificuldades em compreender e dominar decimais (Stacey, \& Steinle, 1998; Glasgow et al., 2000; Rittle-Johnson et al., 2001). De fato, existem estudos que mostram que até mesmo adultos e professores de matemática em formação possuem problemas com números decimais (Putt, 1995; Stacey et al., 2001).

Uma possível forma de remediar esta situação é trabalhar o conceito de decimais utilizando técnicas pedagógicas que não são normalmente utilizadas pelos professores. Uma destas técnicas é a utilização de exemplos incorretos. Estes exemplos são raramente usados em sala de aula ou empiricamente investigados como ferramenta pedagógica para o ensino de matemática. Por outro lado, alguns pesquisadores têm argumentado que confrontar os alunos com erros matemáticos pode criar situações muito benéficas à aprendizagem; particularmente quando os alunos estão suficientemente preparados para lidar com erros (Grosse \& Renkl, 2007; Tsovaltzi et al., 2010).

Com o objetivo de desenvolver um conteúdo Web diferenciado para o ensino de decimais foi desenvolvido materiais instrucionais que podem ser acessados via Internet e que facilitam a aprendizagem por reflexão através da manipulação e explicação de erros. Para avaliar os efeitos da aprendizagem com exemplos incorretos no domínio dos números decimais, um estudo empírico com 429 alunos da sexta, sétima e oitava séries de duas escolas públicas foi realizado nos EUA.

O trabalho está dividido da seguinte forma. A seção 2 apresenta os trabalhos correlatos na área de exemplos incorretos. A seção 3 relata o desenvolvimento de conteúdo Web que utiliza os exemplos incorretos para ensinar decimais. A seção 4 descreve o experimento e os resultados obtidos. E finalmente, na seção 5 temos as conclusões da pesquisa realizada.

\section{Exemplos Incorretos}

O "exemplo incorreto" (erroneous example) é uma solução passo-a-passo para um problema no qual um ou mais passos estão incorretos. Algumas pesquisas e teorias em 
educação matemática que exploram o fenômeno da aprendizagem fornecem algumas evidências de que estudar através de erros pode contribuir para a construção do conhecimento matemático (Borasi, 1987; 1996). Por exemplo, Borasi argumenta que a educação matemática poderia se beneficiar mais da discussão de erros em sala de aula, pois essa prática pedagógica incentiva o pensamento crítico e proporciona novas oportunidades para se resolver problemas baseado na reflexão e na investigação.

Os relatórios da OECD publicados em 2001 e 2010, mostram que os alunos do oriente (e.g. China, Singapura, Japão e Coréia) tem uma performance muito melhor nas avaliações internacionais se comparado à maioria dos países do ocidente (incluindo Brasil e os EUA). Uma das diferenças fundamentais na abordagem pedagógica apontada nestes relátorios foi o método de ensino baseado na apresentação de soluções incorretas. Nesta situação os educadores trabalham para facilitar a discussão entre os alunos para que estes possam localizar e corrigir erros nas soluções apresentadas (OECD, 2001; 2010). Essas e outras análises discutidas nestes relatórios sugerem que o confronto e a reflexão sobre erros podem levar à erradicação dos mesmos, semelhante ao que tem sido demonstrado em pesquisas relacionadas à aquisição de conceitos errôneos (misconceptions) (Bransford, Brown, Armando, 1999).

Por outro lado, muitos professores são ambivalentes (ou até mesmo hostis) quanto à discussão de erros em sala de aula (Tsamir \& Tirosh, 2003). Acredita-se que a exposição de exemplos incorretos pode levar o aluno a fixar os erros e, portanto, o seu uso deveria ser evitado. Alguns educadores que defendem o uso de exemplos incorretos indicam que essa reação é resultado do behaviorismo que teve grande impacto nos métodos educacionais do século passado (Isotani et al., 2011). As experiências behavioristas de Skinner mostram que a falta de uma penalidade quando um sujeito exibir um comportamento indesejável leva a repetição deste comportamento no futuro (Skinner, 1968).

Assim, as questões sobre como utilizar (ou não utilizar) exemplos incorretos para melhorar a aprendizagem de matemática ainda possuem respostas controversas. Para averiguar o real impacto desta ferramenta pedagógica, estudos empíricos controlados vêm sendo realizados para verificar os efeitos dos exemplos incorretos na aprendizagem. Por exemplo, o estudo no domínio das probabilidades conduzido por Grosse \& Renkl (2007) com 118 estudantes universitários alemães identificou que a apresentação de exemplos corretos e incorretos para um mesmo problema ajudam alunos com bom desempenho escolar a aprender e fixar melhor os conceitos se comparado à apresentação de apenas exemplos corretos. Contudo, para alunos com baixo desempenho escolar, a apresentação de apenas exemplos corretos foi mais benéfica. Em outro estudo com 87 alunos da $3^{\text {a }}$ e $4^{\text {a }}$ séries Siegler (2002) verificou que alunos que refletiram sobre exemplos corretos e incorretos aprenderam mais do que aqueles alunos que refletiram apenas sobre exemplos corretos ou aqueles que refletiram sobre suas próprias respostas. De acordo com Siegler, os exemplos corretos ajudam a fixar os conceitos corretos e os exemplos incorretos ajudam a enfraquecer os conceitos errôneos que os alunos possuem.

Além dos trabalhos citados, outros resultados que demonstram os benefícios da aprendizagem com exemplos incorretos foram publicados mais recentemente (Kopp, Stark \& Fischer, 2008; Tsovaltzi et al. 2010). Contudo, nenhum destes trabalhos desenvolveu material didático que pode ser adicionado em ambientes Web de ensino para facilitar e incentivar o uso de exemplos incorretos como prática pedágogica. 
Assim, existem grandes oportunidades para desenvolver conteúdo para facilitar o processo de ensino-aprendizagem utilizando essa abordagem.

\section{Desenvolvimento de Exemplos Incorretos no Domínio de Decimais}

Para criar exemplos incorretos que tem o potencial de suprir as dificuldades dos alunos no domínio de decimais é necessário identificar os erros mais comuns cometidos pelos alunos durante o processo de aprendizagem. Por exemplo, é frequente os alunos tratarem números decimais como se fossem números inteiros. Ou seja, para esses alunos 0.25 é maior que 0.7 , pois 25 é maior que 7 . Alguns estudantes também acreditam que adicionar zeros ao final de um número decimal aumenta o seu valor. Ou seja, 0.2 é menor que 0.20. Estes conceitos errôneos (misconceptions) interferem na compreensão de decimais e se manifestam de diversas maneiras (Stacey \& Steinle, 1998).

Para identificar os conceitos errôneos mais comuns, foi conduzido uma extensa revisão bibliográfica na área de ensino de decimais, abrangendo 42 artigos publicados com início em 1928 (Brueckner, 1928; Graeber \& Tirosh, 1988; Resnick, 1989; Hiebert, 1992; Irwin, 2001; Stacey \& Steinle, 1998). Descobriu-se que muitos conceitos errôneos no domínio dos decimais estão relacionados com o conhecimento prévio do aluno. Para categorizar e criar grupos de conceitos errôneos foram utilizados dados quantitativos disponibilizados pelos trabalhos analisados. Finalmente, uma taxonomia contendo 45 nós foi desenvolvida.

A taxonomia categoriza os conceitos errôneos de acordo com o conhecimento prévio associado ao erro exteriorizado pelo aluno durante a resolução de um problema com decimais. Por exemplo, muitos alunos que estudaram frações antes de aprender decimais acreditam que decimais mais curtos são maiores que decimais mais compridos. Ou seja, 0.3 é maior que 0.367 . Isso ocorre porque os alunos acreditam que 0.3 é equivalente a $1 / 3$ ( 1 dividido por 3 ) e 0.367 é equivalente a 1/367 (1 dividido por 367 ) e, portanto, $0.3>0.367$. Em contrapartida, os alunos que só foram expostos a números inteiros são mais propensos a acreditar que os decimais mais compridos são maiores que decimais mais curtos. A taxonomia permite fazer essa diferenciação e, também, inclui conceitos errôneos relacionados às operações com decimais (e.g. soma e multiplicação).

A organização e classificação de conceitos errôneos oferece diversos benefícios para professores e desenvolvedores de conteúdo, pois é possível desenvolver exemplos e exercícios para lidar com as diferentes dificuldades encontradas pelos alunos durante $o$ processo de aprendizagem de decimais. Além disso, é possível também verificar quais conceitos errôneos são mais frequentes entre os alunos. Segundo nossa análise, sete conceitos errôneos correspondem pela maioria dos erros cometidos pelos alunos (Isotani et al., 2011). Entre eles estão:

- Decimais tratados como números inteiros. Este é o erro mais frequente cometido pelos alunos e está associada a outros conceitos errôneos. Os alunos acreditam que decimais devem ser tratados como números inteiros e, portanto, ignoram o ponto decimal (Rittle-Johnson et al, 2001).

o Exemplo: 0.07 é igual a 7.

- Decimais mais compridos são maiores. Neste caso, os alunos acham que podem comparar números decimais, contando os dígitos após o ponto decimal. Os decimais que tiverem mais dígitos são os maiores (Bell et al., 1981). 
oo Exemplo: 0.25 é maior do que 0.7 , pois 0.25 tem 2 dígitos após o ponto decimal e 0.7 tem apenas um dígito.

- Decimais mais curtos são maiores. Os alunos usam o conhecimento sobre frações para lidar com decimais. Assim, ao comparar números decimais o decimal com maior número depois da vírgula é o menor (Stacey \& Steinle, 1998).

oExemplo: 0.4 é maior que 0.567 , pois 0.4 é a fração representada por $1 / 4$ e 0.567 é a fração representada por $1 / 567$.

- Decimais menores que 1 são números negativos. Os alunos acreditam que decimais começando com um zero são menores do que zero (geralmente os alunos não aprenderam o conceito de números negativos) (Putt, 1995).

oExemplo: 0.25 é menor que zero

- Decimais são dois números inteiros separados por um ponto. Os alunos acreditam que os números do lado esquerdo do ponto decimal são completamente separados dos números do lado direito. Assim, ao somar dois números decimais a resposta será incorreta se existir a necessidade de "levar" um dígito para a próxima coluna à esquerda do ponto decimal (Irwin, 2001).

oExemplo: $1.5+3.9=4.14$

- Multiplicação faz o número ficar maior. Um dos conceitos errôneos mais comuns com operações decimais. Os alunos acreditam que o produto da multiplicação é sempre maior do que seus fatores (Fischbein et al, 1985)

oExemplo: 5 multiplicado por 0,5 é maior que 5

- Divisão faz o número ficar menor. Os alunos acreditam que o quociente de uma divisão é sempre menor do que o número mais à esquerda da operação (dividendo) (Graeber \& Tirosh, 1988).

oExemplo: 3 dividido por 0,5 é menor que 3

Para oferecer aos alunos a oportunidade de praticar suas habilidades cognitivas e metacognitivas (em vez de praticar procedimentos) utilizando exemplos incorretos foram desenvolvidos materiais que facilitam a aprendizagem em três etapas. Primeiro, o aluno compara a solução correta com a incorreta. Segundo, o aluno tenta compreender por que uma determinada solução está incorreta. E terceiro, o aluno tenta explicar como corrigir a solução incorreta. Esta abordagem permite que os alunos aprendam e fiquem cientes dos erros comuns resultantes de conceitos errôneos. Acredita-se também que o material desenvolvido incentiva o pensamento crítico e motiva a reflexão e o questionamento. Este processo composto por três etapas também é consistente com a teoria de Stellan Ohlsson (1996) sobre a aprendizagem baseada em erros. Em sua teoria Ohlsson define que, para aprender com erros, é preciso perceber que algo está errado. Então, é preciso entender qual o tipo do erro. E, finalmente, usar o conhecimento correto para corrigir o problema.

A Figura 1 mostra um exemplo incorreto desenvolvido utilizando o conceito errôneo "Decimais mais compridos são maiores". Iniciamente, o aluno vê apenas a declaração do problema no canto superior-esquerdo e a solução incorreta no centrosuperior da figura. Ao apertar um botão, a solução correta do problema aparece do lado direito da solução incorreta. Nesta situação, os alunos podem comparar as duas soluções 
e tem a oportunidade de responder duas questões. A primeira delas tem como objetivo fazer o aluno refletir sobre a solução incorreta e reconhecer qual é o problema (conceito errôneo). A segunda questão é formulada para motivar o aluno a utilizar seus conhecimento para corrigir a solução incorreta e, ainda, contribuir para que outros alunos também consigam entender o exemplo. Para responder as questões o aluno constrói sentenças utilizando um menu múltipla-escolha. Caso a sentença seja construída corretamente sua cor é modificado para verde. Caso contrário, sua cor é alterada para vermelha e o aluno tem a chance de construí-la novamente.

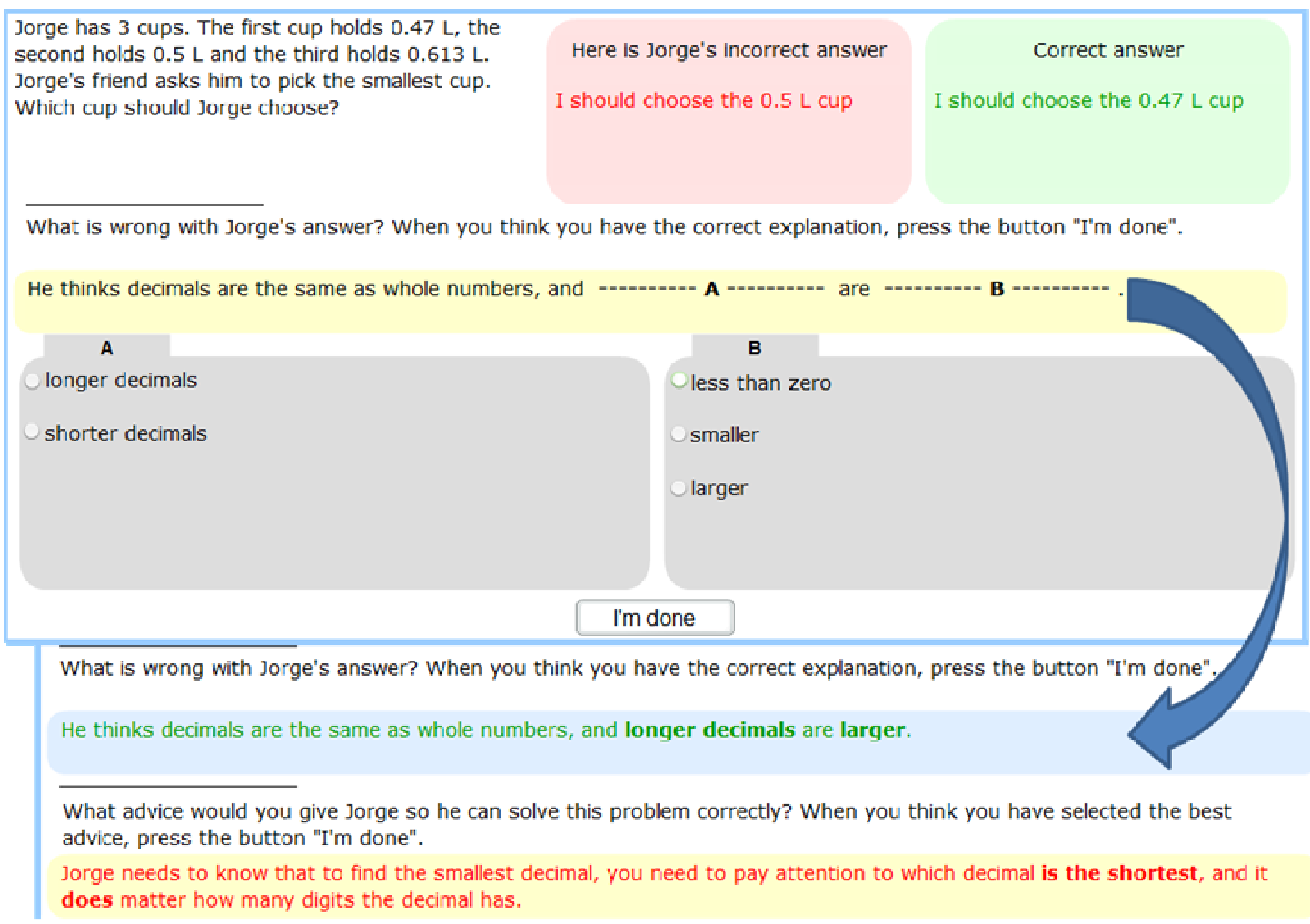

Figura 1. Um exemplo incorreto construido utilizando o conceito errôneo "Decimais mais compridos são maiores".

A tecnologia aplicada para a criação destes exemplos é a mesma utilizada para o desenvolvimento de sistemas tutores inteligentes, conhecida como CTAT - Cognitive Tutor Authoring Tools (Aleven et al., 2006). Esta tecnologia permite criar conteúdo Web que monitora o comportamento do aluno durante a resolução de exercícios. Assim, é possível verificar rapidamente se a resposta do aluno esta correta ou não e, também, analisar o comportamento do aluno ao resolver o problema. Esse comportanto (e.g. motivado ou desmotivado) pode influenciar muito a performance do aluno e, portanto, ter essa informação é fundamental para diagnosticar dificuldades.

Para criar o material didático utilizamos 3 passos: (1) Desenvolvimento da interface; (2) Criação do modelo especialista utilizando um grafo de estados; (3) Identificação de erros típicos e sua inclusão no grafo de estados. O CTAT oferece um conjunto de ferramentas que permite o usuário (e.g. professor) criar a interface do material em Java ou Flash rapidamente. Neste trabalho foi utilizado o Flash devido a praticidade de desenvolver conteúdo interativo e o apelo gráfico que essa tecnologia proporciona. O modelo especialista reflete o conhecimento que o aluno irá adquirir durante a interação com o material (conhecimento desejado). Esse modelo é representado no CTAT como um grafo de estados. Por exemplo, a Figura 2 apresenta 
parte do grafo de estados para o problema da Figura 1. A medida que o aluno interage com o material, é possível estimar o seu nível de aprendizagem. Assim, quando uma questão é respondida adequadamente, o CTAT modifica o estado do aluno indicando que o conceito desejado foi adquirido. Na Figura 2, as setas em negrito indicam o caminho percorrido pelo aluno e o estado em negrito (state 5) indica o estado atual do aluno em relação ao modelo especialista. Os rótulos associados as setas do grafo indicam qual foi a interação realizada pelo aluno e qual é a interação desejada descrita no modelo. Por fim, são adicionados estados relacionados aos erros típicos cometidos pelos alunos para que seja possível tratá-los de maneira adequada e personalizada. Por exemplo, quando um aluno utiliza o material desenvolvido, é possível verificar quais são seus conceitos errôneos e qual a melhor forma de lidar com eles em atividades posteriores. Todas as informações sobre a interação do aluno com o material são armazenadas em logs e disponibilizadas em um banco de dados educacional, conhecido como datashop (https://pslcdatashop.web.cmu.edu/index.jsp), para análise estatística e a busca por padrões de interação/dificuldade utilizando técnicas de mineração de dados.

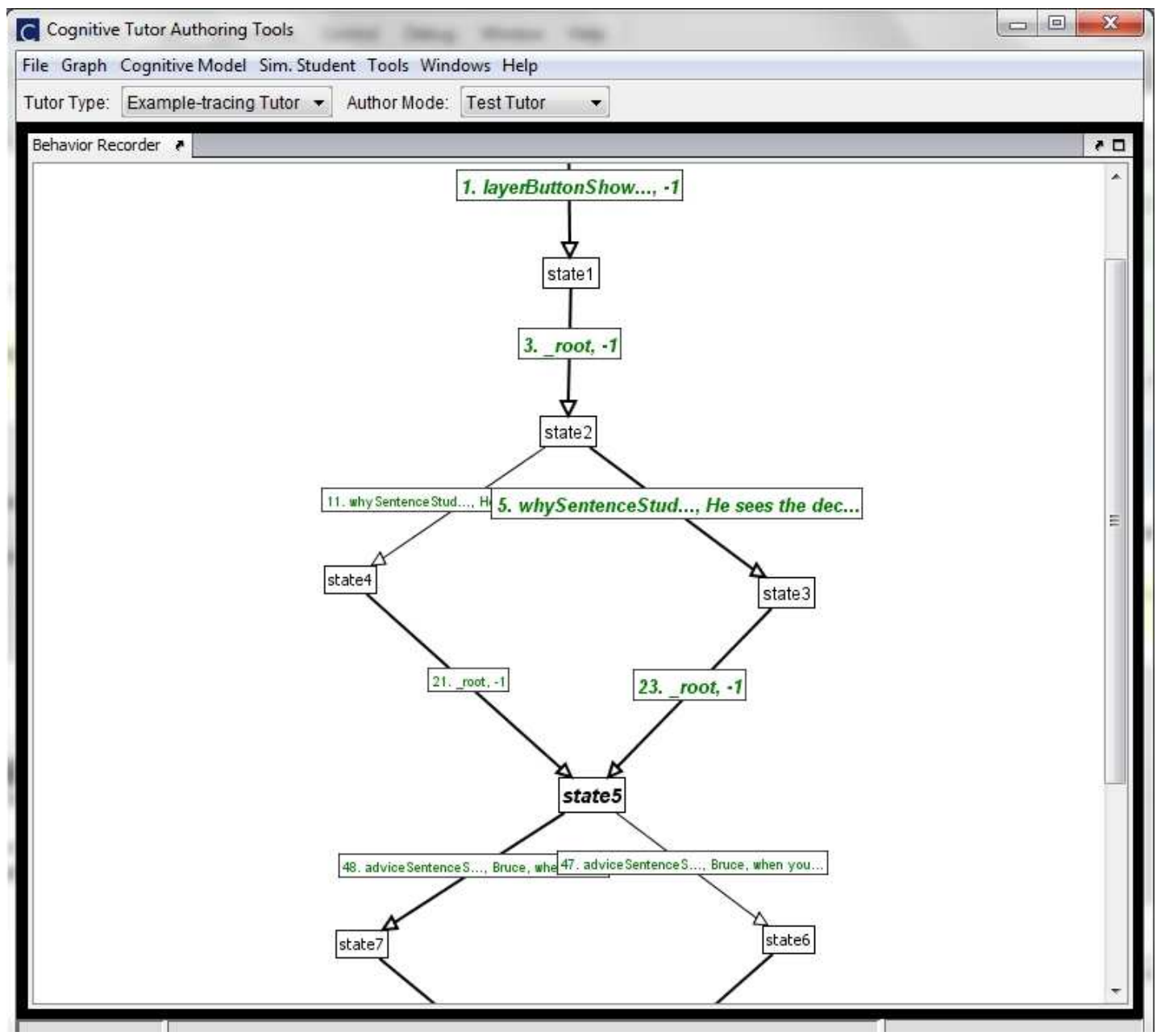

Figura 2. Grafo de estados para o problema apresentado na Figura 1.

Além do exemplo apresentado na Figura 1, dezenas de outros foram desenvolvidos utilizando os sete conceito errôneos apresentados nesta seção. 


\section{Experimento: Avaliação do Conteúdo Desenvolvido}

Devido ao controverso uso do exemplos incorretos no ensino realizou-se um estudo com 429 alunos que estudavam nas sextas, sétimas e oitavas séries de duas escolas públicas do estado da Pensilvânia nos EUA. Estes alunos foram divididos em três grupos. O primeiro grupo recebeu apenas exemplos corretos (EC). O Segundo grupo recebeu apenas exemplos incorretos (EI). E o terceiro grupo recebeu apenas exercícios para resolver (ER).

Criou-se um conjunto de 100 exercícios de teste que foram distribuídos em três provas distintas: o pré-teste, pós-test e o segundo pós-teste. Os testes foram balanceados para terem os mesmos tipos de problemas, com dificuldades semelhantes. Todo o material desenvolvido, incluindo os testes, os exemplos corretos e incorretos e os exercícios foram inseridos em um ambiente virtual de ensino chamado MathTutor (https://mathtutor.web.cmu.edu/). Ao se cadastrar no ambiente, os alunos eram automaticamente incluídos em um dos três grupos. No início do experimento, realizavam o pré-teste. Depois, um conjunto de 30 problemas era oferecido para que os alunos estudassem. Dependendo do grupo em que o aluno se encontrava esses 30 problemas eram exemplos incorretos (conforme apresentado na Figura 1), exemplos corretos, ou apenas exercícios para se resolver. Ao final destes 30 problemas o pós-teste era aplicado e após uma semana o segundo pós-teste era realizado. No total, os alunos utilizaram o ambiente por uma semana durante o período das aulas de matemática.

A tabela 2 mostra a performance dos alunos em cada grupo para cada um dos testes. Observou-se uma melhora sutil na performance dos alunos em todos os grupos. Para verificar se esse resultados são estatisticamente significantes o teste $t$ foi utilizado. Os resultados revelaram que ocorreu melhora significativa em todas as condições entre o pré-teste e o pós-teste $t(254)=-.7928, p<.001$. Além disso, através da análise dos logs armazenados pelo sistema, foi possível identificar os principais erros cometidos pelos alunos durante a resolução dos testes. Para cada problema, a resposta do aluno foi armazenada e rotulada como correta, incorreta devido ao uso de conceitos errôneos ou incorreta devido a fatores desconhecidos. Verificou-se que aproximadamente $31 \%$ dos erros cometidos no pré-teste foram resultados diretos de um ou mais conceitos errôneos apresentados na seção 3. Enquanto que após as atividades com o uso de exemplos incorretos a porcentagem caiu para 24\%. Estes resultados sugerem que o uso de exemplos incorretos oferece benefícios ao aprendizado de decimais, confirmando os resultados obtidos por outros pesquisadores em outras áreas da matemática.

Tabela 1. Média dos alunos (M) e o desvio padrão (DP)

\begin{tabular}{lcccccc} 
& & & & \multicolumn{3}{c}{$\begin{array}{c}\text { Segundo } \\
\text { pós-teste }\end{array}$} \\
\cline { 2 - 7 } Grupo & M & DP & M & DP & M & DP \\
\hline ER & $\mathbf{0 . 5}$ & 0.15 & $\mathbf{0 . 5 7}$ & 0.16 & $\mathbf{0 . 5 6}$ & 0.17 \\
EC & $\mathbf{0 . 5 4}$ & 0.17 & $\mathbf{0 . 6 1}$ & 0.18 & $\mathbf{0 . 6}$ & 0.19 \\
EI & $\mathbf{0 . 5 3}$ & 0.16 & $\mathbf{0 . 5 9}$ & 0.18 & $\mathbf{0 . 5 8}$ & 0.19 \\
\hline
\end{tabular}




\section{Conclusão}

Este trabalho teve como objetivo desenvolver conteúdo Web para estudar o uso de exemplos incorretos no ensino de decimais. Para atingir tal objetivo foi conduzido um estudo aprofundado sobre os principais problemas durante a aprendizagem de decimais e a classificação destes problemas em uma taxonomia de conceitos errôneos. Baseado nestes conceitos errôneos, criou-se diversos exemplos incorretos que foram incluídos em um ambiente virtual de aprendizagem.

Para verificar os benefícios deste material ao ensino de decimais foi conduzido um estudo com 429 alunos das sextas, sétimas e oitavas séries de duas escolas públicas dos EUA. Os resultados obtidos sugerem que exemplos incorretos são tão benéficos (e às vezes até mais benéficos) quanto outros materiais comuns utilizados em sala de aula, como exemplos corretos e exercícios. Embora os resultados obtidos sejam positivos, ainda é precoce concluir que o uso de exemplos incorretos é sempre benéfico ao aprendizado. Como o experimento realizado foi de apenas uma semana, em estudos futuros pretende-se verificar o impacto do uso contínuo dos exemplos incorretos quando utilizados por um longo período de tempo. É também objetivo futuro dos autores disponibilizarem todo conteúdo desenvolvido de forma gratuita para que qualquer professor ou aluno tenha acesso a esse tipo de material diferenciado.

\section{References}

Aleven, V., McLaren, B. M., Sewall, J., \& Koedinger, K. R. The Cognitive Tutor Authoring Tools (CTAT): Preliminary Evaluation of Efficiency Gains. In International Conference on Intelligent Tutoring Systems. LNCS 4053, pp. 6170. Springer-Heidelberg, 2006.

Bell, A. Swan, M. \& Taylor, G. Choice of operations in verbal problems with decimal numbers. Educational Studies in Mathematics, 12, pp. 399-420, 1981.

Borasi, R. Exploring mathematics through the analysis of errors. For the Learning of Mathematics: An International Journal of Mathematics Education, 7(3), pp 28, 1987.

Borasi, R. Reconceiving mathematics instruction: A focus on errors. Norwood, NJ: Ablex, 1996.

Bransford, John, Brown, Ann L., \& Cocking, Rodney R. How People Learn: Brain, Mind, Experience, and School. Washington, D.C.: National Academy Press, 1999.

Brueckner, L.J. Analysis of Difficulties in Decimals. Elementary School Journal, 29, 32-41, 1928.

Fischbein, E., M. Deri, M. Nello, \& M. Marino The role of implicit models in solving verbal problems in multiplication and division. Journal of Research in Mathematics Education, 16, pp 3-17, 1985.

Glasgow, R., Ragan, G.; Fields, W.M.; Reys, R. \& Wasman, D. The decimal dilemma. Teaching Children Mathematics, vol. 7, pp. 89-93, 2000.

Graeber, A. \& Tirosh, D. Multiplication and division involving decimals: Preservice elementary teachers' performance and beliefs. Journal of Mathematics Behavior, 7, 263-280, 1988. 
r

Grosse, C.S. \& Renkl, A. Finding and fixing errors in worked examples: Can this foster learning outcomes? Learning and Instruction, 17(6), pp. 612-634, 2007.

Hiebert, J. Mathematical, Cognitive, and Instructional Analyses of Decimal Fractions. Chapter 5 in Analysis of arithmetic for mathematics teaching, pp 283322. Lawrence Erlbaum, 1992.

Irwin, K.C. Using everyday knowledge of decimals to enhance understanding. Journal for Research in Mathematics Education, 32(4), pp. 399—420, 2001.

Isotani, S., Adams, D., Mayer, R.E., Durkin, K., Rittle-Johnson, B., \& McLaren, B.M. Can erroneous examples help middle-school students learn decimals?. In European Conference on Technology Enhanced Learning. LNCS, Springer-Heidelberg, 2011.

Kopp, V., Stark, R., \& Fischer, M. R. Fostering diagnostic knowledge through computer-supported, case-based worked examples: Effects of erroneous examples and feedback. Medical Education, vol. 42, pp. 823-829, 2008.

Mayer, R. E., Sims, V. \& Tajika, H. A comparison of how textbooks teach mathematical problem solving in Japan and the United States. American Educational Research Journal, 32(2), pp. 443-460, 1995.

Melis, E. Design of erroneous examples for ActiveMath. In Proceedings of the International Conference on Artificial Intelligence in Education, pp. 451-458. IOS Press, 2005.

OECD. Knowledge and Skills for Life. First Results from PISA 2000, Paris, 2001.

OECD. Results from PISA 2010, Paris, 2010.

Ohlsson, S. Learning from performance errors. Psychological Review, Vol. 103, No. 2 , 241-262, 1996.

Putt, I. J. Preservice teachers ordering of decimal numbers: When more is smaller and less is larger!. Focus on Learning Problems in Mathematics, 17(3), pp. 1-15, 1995.

Resnick, L. B., Nesher, P., Leonard, F., Magone, M., Omanson, S., \& Peled, I. Conceptual bases of arithmetic errors: The case of decimal fractions. Journal for Research in Mathematics Education, 20(1), pp. 8-27, 1989.

Rittle-Johnson, B., Siegler, R.S., \& Alibali, M.W. Developing conceptual understanding and procedural skill in mathematics: An iterative process. Journal of Educational Psychology, 93(2), 346-362, 2001.

Siegler, R.S. Microgenetic studies of self-explanation. In N. Granott and J. Parziale (eds). Microdevelopment, Transition Processes in Development and Learning, pp. 31-58. Cambridge University Press, 2002.

Skinner, B. F. The Technology of Teaching. New York: Appleton-Century-Crofts, 1968

Stacey, K., Helme, S., Steinle, V., Baturo, A., Irwin, K., \& Bana, J. Preservice teachers' knowledge of difficulties in decimal numeration. Journal of Mathematics Teacher Education, 4(3), 205--225, 2001. 
Stacey, K. \& Steinle, V. Refining the classification of students' interpretations of decimal notation. Hiroshima Journal of Mathematics Education, vol. 6, pp. 4969, 1998.

Resnick, L. B., Nesher, P., Leonard, F., Magone, M., Omanson, S., \& Peled, I. Conceptual bases of arithmetic errors: The case of decimal fractions. Journal for Research in Mathematics Education, 20(1), 8-27, 1989.

Tsamir, P. \& Tirosh, D. In-service mathematics teachers' views of errors in the classroom. In International Symposium: Elementary Mathematics Teaching, 2003.

Tsovaltzi, D., Melis, E., McLaren, B.M., Meyer, A-K., Dietrich, M. \& Goguadze, G. Learning from erroneous examples: When and how do students benefit from them? In European Conference on Technology Enhanced Learning, LNCS vol. 6383, pp. 357-373. Springer-Heidelberg, 2010. 